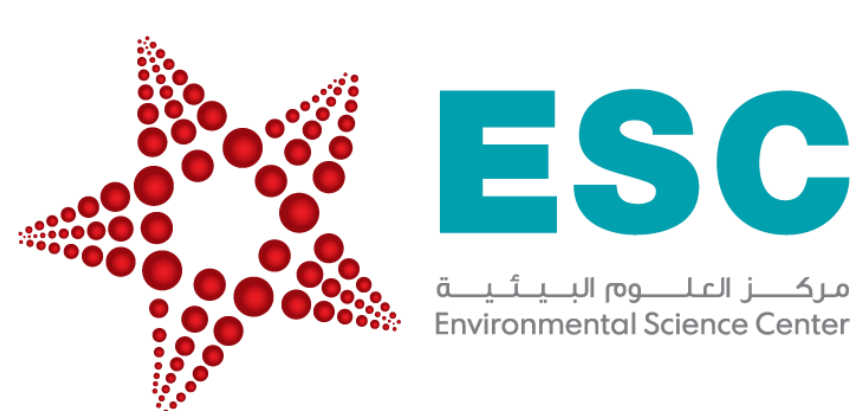

\title{
Coral Research and Nursery Farm Project
}

\author{
P. Range ${ }^{1}$, B. Giraldes ${ }^{1}$, J. Al-Khayat ${ }^{1}$, M. Romeo ${ }^{1}$, N. Chacko ${ }^{1}$, M. Chatting ${ }^{1}$, A. Alashwal ${ }^{1}$, N. Al-Omari ${ }^{1}$, K. Sarhan ${ }^{1}$, S. Saliba ${ }^{1}$, \\ H. Al-Mohannadi², K. Bashir², M. Abdulla², H. Al-Kuwari ${ }^{1}$
}

\section{1 - Environmental Science Center (ESC), Qatar University; 2 - Qatargas Operating Company Limited}

\section{Introduction}

In the framework of the Coral Management Plan for the North Field Expansion Project (NFE) and North Field Production Sustainability Project (NFPS), Qatargas has partnered with the ESC to develop the first land-based coral nursery in Qatar. This nursery plan includes the extraction of 1000 corals colonies from the NFPS and NFE pipeline corridors, north of Ras Laffan, transport to the nursery facility, rehabilitation under controlled conditions, fragmentation, out-plantation and monitoring of the fragments to carefully selected recipient sites, which include natural and artificial reef habitats. The coral propagation methods used in this project (fragmentation and micro-fragmentation) have, so far, been mainly tested with a restricted number of branching coral species, mostly in in-situ nurseries. Our project is among the first to apply this type of approach to reef restoration in the Arabian Gulf.

\section{Objectives}

- This project will increase the Technological Readiness Level (TRL) of the procedures and facilities for asexual propagation of local coral species in a land-based nursery (fragmentation and husbandry) and successful reintroduction (outplant) to natural or artificial marine habitats.

- We estimate that up to 10,000 coral fragments will be outplanted and monitored over a five year period.

\section{Methods}

The first two batches of 200 corals (batch1 - 1500 fragments, batch 2 - 1200 fragments) were extracted in March and outplanted in April 2021. Both recipient sites are located in the North of Sheraoha Island, within $1 \mathrm{~km}$ of each other. The main stages of this process are illustrated in Fig. 1 and summarized here:

- The coral colonies were extracted from the pipeline corridors by SCUBA divers using small hammers and chisels and transported in pools with running seawater to the nursery facility, located at the Aquatic \& Fisheries Research Center (AFRC) at Ras Matbakh (Fig.1A) On arrival to the AFRC the corals are transferred to $500 \mathrm{~L}$ holding tanks (Fig.B), individually labeled and photographed (Fig.1C)

- The colonies are fragmented using a wet bandsaw. Different sizes, between 1.5 and $10 \mathrm{~cm}$ diameter are used, to assess the effect of fragment size on survival and growth (Fig.1D).

- The fragments are attached to aragonite tiles using marine epoxy, assembled in arrays and kept in recirculating raceways equipped with sump, biological filter, protein skimmer, wave makers, UV sterilizer and automated control of lighting and temperature (Fig.1E).

- The day prior to each outplant the fragments are organized in labeled trays and storage boxes and returned to the $500 \mathrm{~L}$ holding tanks (Fig.1F-H).

- Early next day the fragments are transported by car to the jetty (Fig.1I) and by speedboat to the QU research Vessel (RV Janan, Fig.1J-K), where they are maintained under running seawater during sailing to the selected recipient site (Fig.1L)

- On the second day the corals are transferred by speedboat to the predefined outplant area where the boxes are carefully lowered to the seafloor and collected by divers (Fig.1M-Q).

Portland cement is mixed with $10 \%$ of microsilica and seawater, to achieve a clay-like texture and lowered to the seafloor in 5 gallon buckets.

- Divers assign the trays with fragments from each colony to a suitable patch of substrate and attach them using a small PVC mold, to contain the cement and avoid excessive sedimentation (Fig.1R-U).

Finally, a numbered tag is attached to the wet cement base, to allow identification of each colony during monitoring (Fig.1V).

- Each of the recipient sites will have at least 8 monitoring events during the project, at months $1,3,6,12,18,24,36$ and 48.

- The first monitoring events were done in April and June 2021.

- The monitoring protocol is based on underwater visual census (UVC) and photoquadrats.

UVCs provide high quality data on attachment success, survival and health status of the corals.

Photoquadrats allow the assessment of coral growth, at 6 months intervals, by measuring changes in coral surface are as well as the identification and quantification of new coral recruits and cover of other functional groups of benthic fauna.

- Environmental data is also being collected, including continuous in-situ logging of seawater temperature and light intensity.

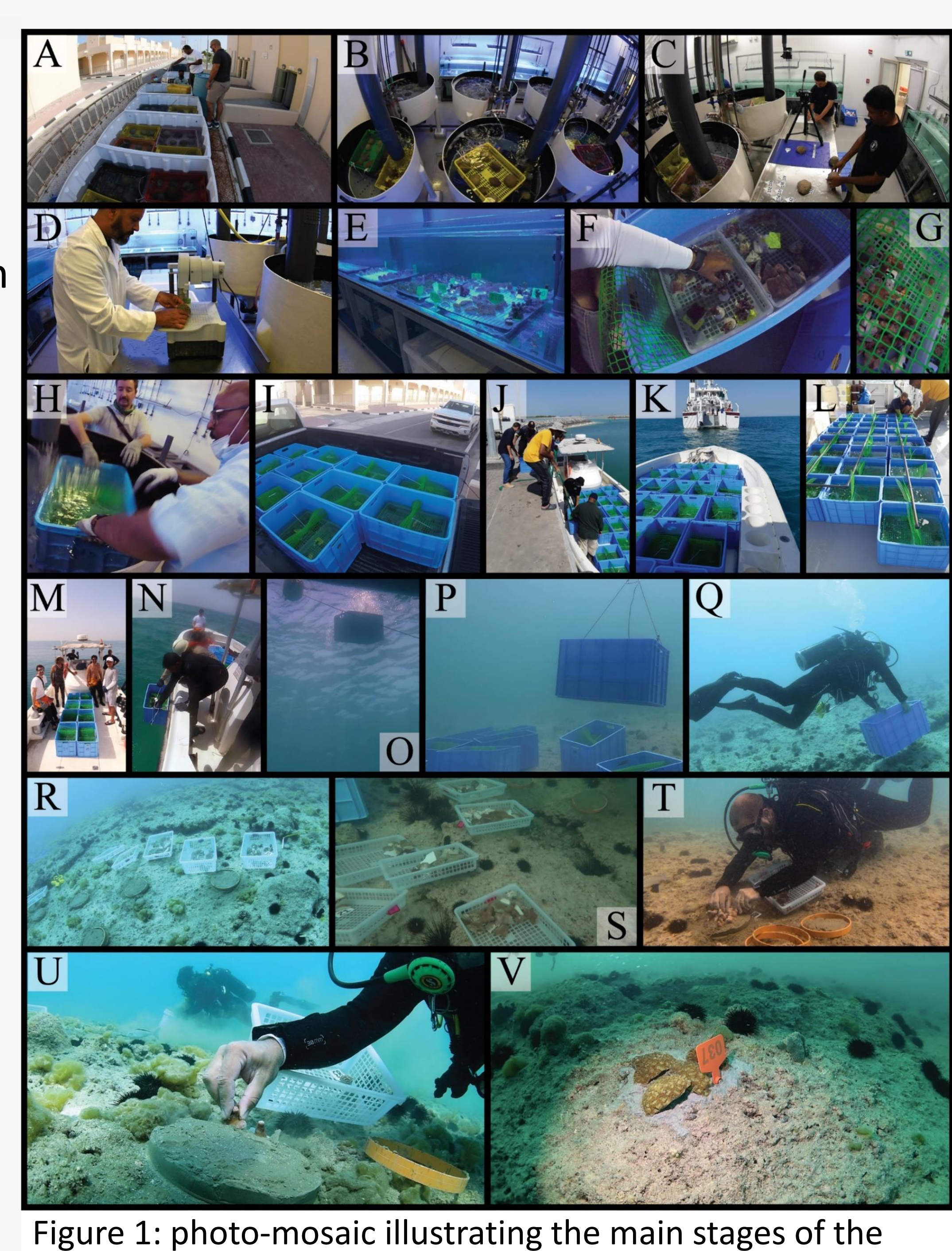
project. See Methods for details

\section{Results}

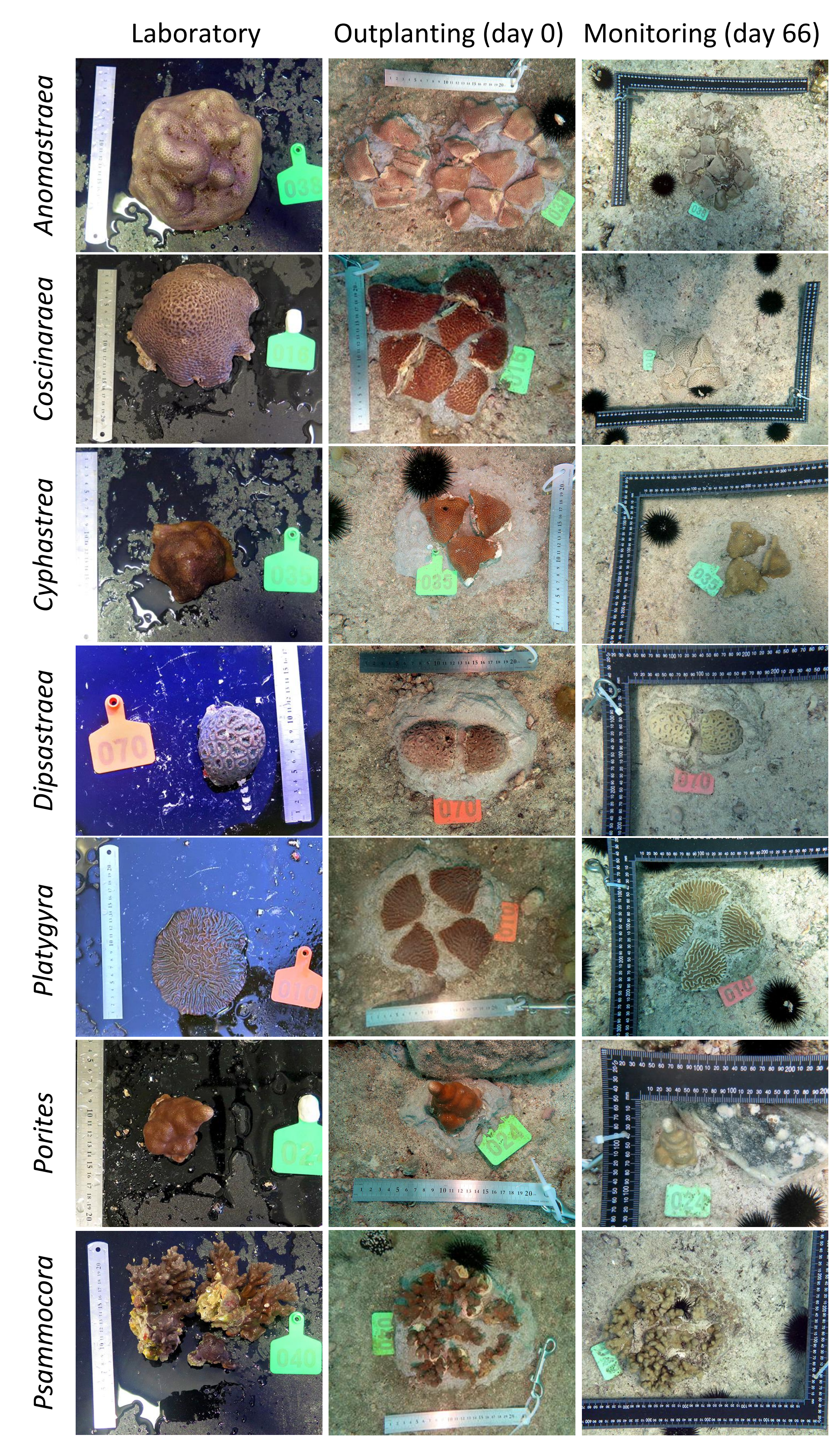

Figure 2: Progression of one colony of each of the seven coral genera tested so far since arrival at the AFRC nursery until the first monitoring.

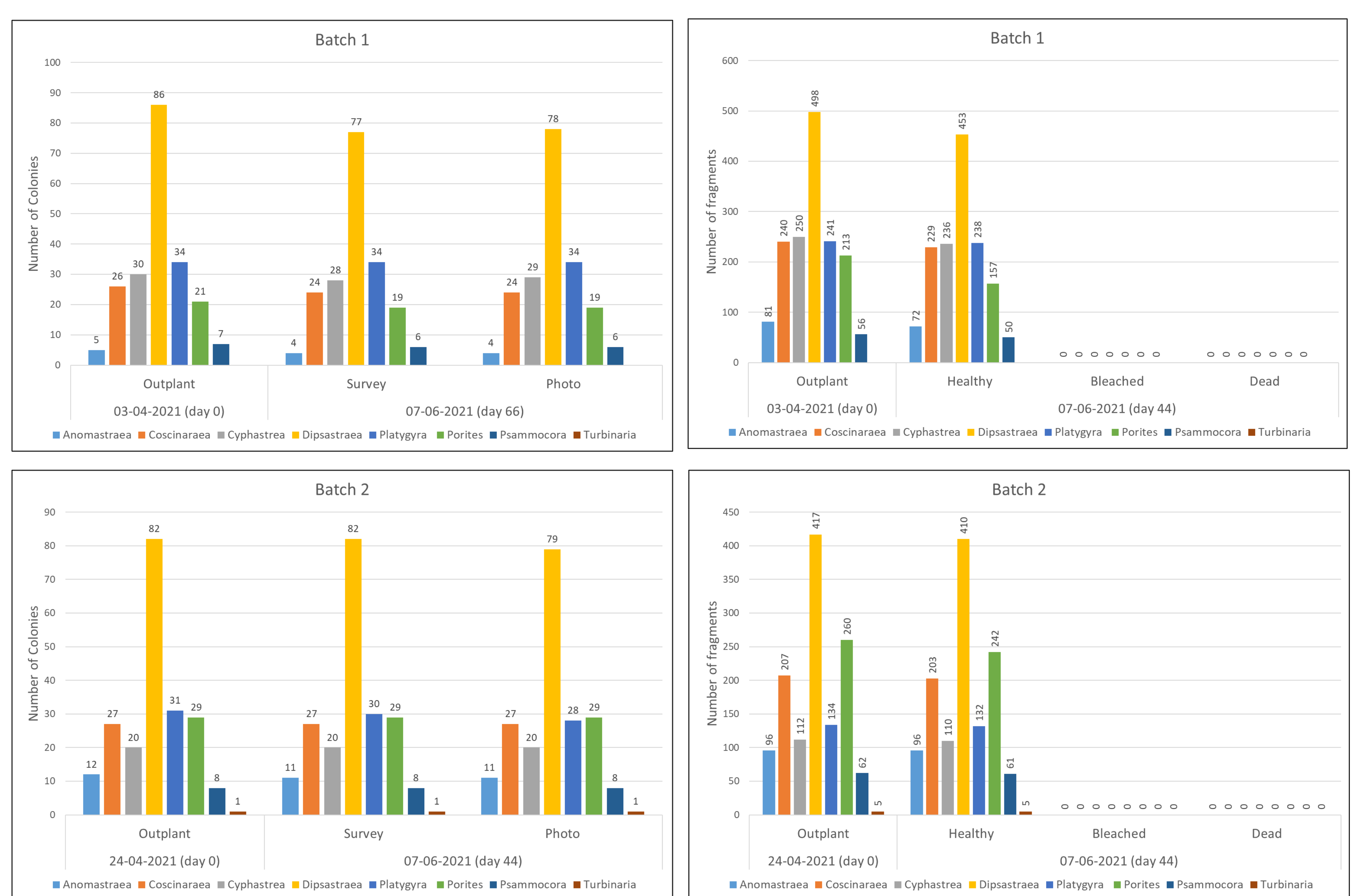

Figure 3: Number of colonies of each coral genus outplanted in each batch and the numbers surveyed and photographed during the last monitoring event (left): Number of fragments of each coral genus outplanted in each batch, survival and health status during the last monitoring event (right)

\section{Conclusions}

- The coral propagation methods (i.e., fragmentation, husbandry and outplanting) used in this project are well established and widely used in coral restoration projects all over the world. We successfully adapted these tools to the extreme marine environment of Qatar and to the local pool of coral species.

The initial monitoring results are encouraging, but the real effectivity of coral restoration can only be evaluated with long-term monitoring. Our five year timeline largely exceeds the global average duration for this type of projects (18 months). - If the long-term survival of our outplants is in line with the global average (50\%) we predict a significant increase in coral cover and coral recruitment at the recipient sites. Developing the capacity to mass produce and successfully deploy coral propagules, in a controlled way, under detailed Standard Operational Procedures, will massively increase the impact, as well as reduce the costs and risks associated with future coral restoration and compensation efforts in Qatar and the Gulf region.

- This Coral Nursey Program has clear potential to lay down the foundations for the establishment of a national/regional coral bank, to be used as a tool for adaptation to climate change and preventing the loss of marine biodiversity. 